Research Article

\title{
How strong was the bottleneck associated to the peopling of the Americas? New insights from multilocus sequence data
}

Nelson J. R. Fagundes ${ }^{1}$ D, Alice Tagliani-Ribeiro ${ }^{2}$, Rohina Rubicz ${ }^{3}$, Larissa Tarskaia ${ }^{4}$, Michael H. Crawford $^{4}$, Francisco M. Salzano ${ }^{1}$ and Sandro L. Bonatto ${ }^{5}$

${ }^{1}$ Departamento de Genética, Universidade Federal do Rio Grande do Sul (UFRGS), Porto Alegre, RS, Brazil.

${ }^{2}$ Fertilitat Centro de Medicina Reprodutiva, Centro Clínico da Pontifícia Universidade Católica do Rio Grande do Sul (PUCRS), Porto Alegre, RS, Brazil.

${ }^{3}$ Division of Public Health Sciences, Fred Hutchinson Cancer Research Center, Seattle, WA, USA.

${ }^{4}$ Laboratory of Biological Anthropology, University of Kansas, Lawrence, KS, USA.

${ }^{5}$ Faculdade de Biociências, Pontifícia Universidade Católica do Rio Grande do Sul (PUCRS), Porto Alegre, RS, Brazil.

\begin{abstract}
In spite of many genetic studies that contributed for a deep knowledge about the peopling of the Americas, no consensus has emerged about important parameters such as the effective size of the Native Americans founder population. Previous estimates based on genomic datasets may have been biased by the use of admixed individuals from Latino populations, while other recent studies using samples from Native American individuals relied on approximated analytical approaches. In this study we use resequencing data for nine independent regions in a set of Native American and Siberian individuals and a full-likelihood approach based on isolation-with-migration scenarios accounting for recent flow between Asian and Native American populations. Our results suggest that, in agreement with previous studies, the effective size of the Native American population was small, most likely in the order of a few hundred individuals, with point estimates close to 250 individuals, even though credible intervals include a number as large as $\sim 4,000$ individuals. Recognizing the size of the genetic bottleneck during the peopling of the Americas is important for determining the extent of genetic markers needed to characterize Native American populations in genome-wide studies and to evaluate the adaptive potential of genetic variants in this population.
\end{abstract}

Keywords: Isolation with Migration model, coalescent analysis, native Siberians.

Received: March 24, 2017; Accepted: May 23, 2017.

\section{Introduction}

Despite many scientific efforts have been made to unveil the peopling of the Americas, several important questions are still elusive (see Salzano, 2007; Goebel et al., 2008; González-José and Bortolini, 2011; O’Rourke, 2011; Marangoni et al., 2014 for recent reviews). Since the classic tripartite hypothesis for the origin of Native Americans proposed by Greenberg et al. (1986), a range of migration theories have been put forward to account for the linguistic, genetic, and morphologic diversity of human populations in the New World (see Marangoni et al., 2014). Concerning genetic data, the analysis of uniparental markers have shown that most genetic diversity in Native Americans derives from a major population expansion after the Last Gla-

Send correspondence to Nelson J. R. Fagundes, Departamento de Genética, UFRGS, Caixa Postal 15053, 91501-970, Porto Alegre, RS, Brazil. E-mail: nelson.fagundes@ ufrgs.br. cial Maximum (LGM) from an ancestral Beringian population (Zegura et al., 2004; Tamm et al., 2007; Achilli et al., 2008, 2013; Fagundes et al., 2008; Mulligan et al., 2008; Bisso-Machado et al., 2011, 2012; Mulligan and Szathmàry, 2017), but also that a single "migration wave" was too simplistic to account for the distribution of rare lineages, especially in North America, in agreement to the wide morphological variation found in Native American populations (e.g.. González-José et al., 2008). Genomic studies based on a wide set of genetic markers have confirmed and extended this finding. A formal model choice procedure based on 401 microsatellite loci found that a model including recurrent gene flow between Siberian and Native American populations provided a better fit to the data compared to a model without gene flow (Ray et al., 2010). In qualitative agreement with this finding, studies based on hundreds of thousands of SNP markers have consistently find evidence of ancient migration links between Native American 
and other Old World populations (Reich et al., 2012; Raghavan et al., 2014, 2015; Rasmussen et al., 2015; Skoglund et al., 2015).

An important parameter in population genetic studies is the effective population size, which can be broadly defined as the size of a simple Wright-Fisher population that undergoes the same amount of random drift as the actual population considered (Charlesworth, 2009). Characterizing the effective population size is instructive for understanding the selection-drift balance - which determines if nearly-neutral alleles behave as deleterious, neutral or adaptive - and of the size of linkage blocks - which is important in mapping studies (Hartl and Clark, 2007; Charlesworth, 2009). Genomic scans for genetic variation in humans have consistently shown that Native American populations are usually the least diverse in the globe (e.g., Prugnolle et al., 2005; Li et al., 2008), but these same studies find a very good correlation between genetic diversity and distance from East Africa. Therefore, the small genetic diversity in Native American populations could simply result from their long distance from East Africa. However, some colonization events may amplify the loss of genetic diversity if they are accompanied by a strong genetic bottleneck, as was probably the case for the huge differences in genetic variation levels between African and non-African populations (e.g., Yu et al., 2002; Long et al., 2009). Was this the case for Native Americans?

The first quantitative approach to infer the effective population size of the founder Native American population was developed by Hey (2005), who did a meta-analysis of nine sequence loci, used a likelihood-based inference and assumed a isolation with migration (IM) population model to suggest an extreme population bottleneck with an effective population size of $\sim 70$ individuals. Since this pioneer work, other groups tried to replicate this result using multilocus autosomal data, with partial success. Kitchen et al. (2008) re-analyzed Hey's dataset, adding mtDNA genomic data under different priors for migration rates and suggested an effective population size ranging from 1,000 to 5,400 individuals. Ray et al. (2010), using a dataset of 401 STRs, estimated an effective founder population size between 42 and 140 individuals (with a median of 87 individuals). Between these two extremes, Fagundes et al. (2007), based on the re-sequencing of 50 short loci, estimated an effective founder size of $\sim 450$ individuals (with a $95 \%$ credible interval (CI) ranging from 71 to 1,280 individuals). Recent autosomal data generated from admixed Latino populations also provided very different figures. Gutenkunst et al. (2009), based on a very large dataset of more than 13,000 SNPs, suggested a value of 800 effective individuals, with a confidence interval between 140 and 1,600 individuals; while Wall et al. (2011), using resequencing data, estimated a bottleneck effective population size not larger than 150 individuals. Gravel et al. (2013) proposed intermediate values of about 514 effective individuals, ranging between 316 and 2,264 individuals.

In this study we generated DNA sequence data from Native American and Siberian individuals for nine autosomal loci totaling about $\sim 17.5 \mathrm{~kb} /$ individual. We also included data from other Asian individuals and used an isolation-with-migration population model to study the pattern of population subdivision and to estimate the effective population size of the first Native American settlers. To our knowledge, this is the first time that this parameter is explicitly estimated using a common set of individuals from Native American populations typed for autosomal sequence data and analyzed under a full-likelihood method. Overall, our results confirm a late Pleistocene split between Siberians and Native Americans, with Asian populations splitting off some thousand years earlier. Our results also corroborate the idea that the Native American founder population underwent a strong bottleneck, though less extreme than previously suggested.

\section{Material and Methods}

\section{Samples and ethics statement}

We selected DNA samples from 10 Native American individuals scattered across Central and South America, representing several different tribal affiliations. More specifically, we used DNA samples of one individual from each of the following populations: Aché (Paraguay), Arara (Brazil), Bribri (Costa Rica), Guatuso (Costa Rica), Guaymi (Costa Rica), Lengua (Argentina), Quechua (Peru), Waiwai (Brazil), Xavante (Brazil), and Zoró (Brazil). The same sampling scheme was applied to Siberian populations, and one individual from each of the following populations was studied: Altai, Aleut, Buryat, Chukchi, Evenki, Even, Itel'men, Kalmyk, Koryak, and Tuva. For a more thorough characterization of the genetic diversity in Asia, 15 individuals from China genetically characterized by Frisse et al. (2001) have been included in the final dataset.

For Native American participants, ethical approval was provided by the Brazilian National Ethics Commission (CONEP Resolution no. 123/98), according to all the ethic practices required at the time. Individual and tribal informed oral consent was obtained from all participants, since they were illiterate, and were obtained according to the Helsinki Declaration. Record was made of the Amerindian leaders and National Indian Foundation (FUNAI) officials consents. The ethic committee approved the oral consent procedure, as well as the use of these samples in population and evolutionary studies. The samples from Siberian populations were collected following the collapse of the Soviet Union. Only verbal informed consent was obtained. This form of consent was given with witnesses present. The verbal informed consent was necessary because of the association of signing documents to political confessions during the days of the USSR. Both the University of 
Kansas Institutional Review Board and NSF approved this alternative method of informed consent.

\section{Molecular markers and methods}

We studied nine noncoding autosomal regions first investigated by Frisse et al. (2001). They correspond to regions 1-5 and 7-10 characterized in the indicated study; and each are about $10 \mathrm{kbp}$ in length, for which 1,000 bp at each end was sequenced. This approach has the advantage to detect possible effects of recombination as there is some distance between the edges of each marker. Following the above-indicated authors (Frisse et al., 2001), each of these two-segment units will be referred as a "locus pair". These regions have been also used in other studies (Voight et al., 2005; Wall et al., 2008; Scliar et al., 2012).

Genomic DNA of all samples was initially subjected to a whole genomic amplification (WGA) using GenomePhi (GE Healthcare), a strategy that is considered adequate for subsequent downstream procedures such as PCR and sequencing (El Sharawy et al., 2012). We then used the WGA product diluted 10x as template for the specific PCR amplifications. For each of the nine regions we designed external and internal primer sets to allow for PCR amplification and sequencing by the Sanger method. The amplification of PCR products were checked in agarose gel stained with GelRed ${ }^{\mathrm{TM}}$, and purified with polyethylene glycol (Dunn and Blattner, 1987), after which they were subjected to automated sequencing in a MegaBACE 1000 machine (GE Helthcare) using the manufacturer's kits and protocols.

\section{Data analysis}

Sequences were assembled using PhredPhrap (Ewing et al., 1998) and visualized in Consed (Gordon et al., 1998) using reference sequences obtained from GenBank to guide the assembly. Heterozygous positions were easily identified by visual inspection. All positions containing singletons were confirmed using independent PCR and sequencing reactions. Haplotypes for each locus were estimated using PHASE 2.1 (Stephens et al., 2001) using five independent runs to check for consistency and convergence.

Basic genetic diversity measures, such as haplotype and nucleotide diversity, neutrality tests (Tajima's D and Fu's $F_{S}$ ), and measures based on F-statistics were performed in the Arlequin 3.5 program (Excoffier and Lischer, 2010). The null hypothesis of intralocus no recombination was evaluated in the DnaSP 5 software (Librado and Rozas, 2009) using the ZZ statistic (Rozas et al., 2001). For each locus, the substitution rate was estimated under the assumption of a lognormal relaxed molecular clock (Drummond $e t$ al., 2006) and assuming for the human-chimpanzee divergence a normal distribution with mean of 6.5 million years (e.g., Macaulay et al., 2005) and standard deviation of 0.3 million years. Substitution rate estimates were performed in the Beast 1.6.5 program (Drummond and Rambaut,
2007). For all loci, the $\mathrm{HKY}+\mathrm{G}+\mathrm{I}$ evolutionary model was assumed with parameters allowed to vary freely.

Two alternative demographic assumptions were tested; first a constant population size model and Bayesian skyline demographic model, in which the gene genealogy of each locus is divided in "epochs" that can have different population sizes (Drummond et al., 2005). The demographic model providing the best fit with the data was selected using Bayes Factors (Kass and Raftery, 1995) estimated in Tracer 1.5 (http://beast.bio.ed.ac.uk/Tracer), which in all cases supported the constant population size model (data not shown). Each analysis was run for 100,000,000 generations, sampling every 1,000 generations, and the first $10 \%$ samples were discarded as burn-in.

Demographic parameters were estimated under the isolation-with-migration population model (IM), as implemented in the IM program (Hey, 2005). In short, the model assumes that moving forward in time, an ancestral population of size $\theta_{\mathrm{A}}$ splits in two sister populations at time $\mathrm{T}$ according to parameter $s$, which varies from 0 to 1 . Thus, one of the descendant populations has a founder population size of $\theta_{\mathrm{A}} s$, while the remaining population has a founder size of $\theta_{\mathrm{A}}(1-s)$. After the split, the two populations are allowed to grow or shrink and they may exchange migrants in an asymmetric way.

Because the migration parameter affects the estimates for the founder population sizes (Kitchen et al., 2008), we used two migration scenarios, the first one assuming that no migration took place after the population split, and the second one assuming a maximum migration value estimated from contemporary European populations, as in Kitchen et al. (2008). The lower limit for population split was set at 15 thousand years ago (kya), based on the archeological record for the Americas, for which some of the oldest sites includes the well accepted Monte Verde, in southern Chile, dated at 14,500 years ago, and Swan Point, in Central Alaska, dated at 14,000 years ago (Goebel et al., 2008). The analysis was run for $5,000,000$ steps sampling every 100 steps. Consistency was checked by running the same settings multiple times using different seeds. To ensure the quality of the estimates, the effective sample size (ESS) for all parameters in all scenarios was higher than 500 .

\section{Results and Discussion}

\section{General results and SNP distribution}

The full alignment of all nine regions in Chinese, Siberian, and Native American samples produced a data matrix consisting of 17,456 bp and 66 SNPs. All generated sequences are available in GenBank under accession numbers KF468820-KF469176. Chinese was the population with the highest number of SNPs, followed by Native American and Siberian (49, 39 and 36, respectively). When Chinese and Siberian samples are merged into an "Asian" metapopulation, the number of SNPs rises to 62 , suggesting 
that these two subgroups are genetically distinct, with Siberians having 13/36 SNPs that were not found in Chinese. The Native American sample had four private SNPs, which were not shared with either Chinese or Siberian samples.

\section{Basic population genetic quantities}

Average values for several common population genetics statistics, together with their standard deviations over loci are presented in Table 1. Similar tables for each locus are available in the Table S1 (Supplementary material). In general, observed heterozygosity $\left(H_{\mathrm{OBS}}\right)$ was lower than expected heterozygosity $\left(H_{\mathrm{EXP}}\right)$, which may be related to the Wahlund effect, that is, a deficit in observed heterozygosity caused by population structure (Hartl and Clark, 2007). This is expected from the sampling scheme adopted in this study, in which, for most populations, we sampled genetics lineages from a single individual from different local populations. In line with this reasoning, the smallest difference between $H_{\mathrm{OBS}}$ and $H_{\mathrm{EXP}}$ was found for the Chinese, which is the geographically more homogeneous group, even though local inbreeding may also play a role in lowering $H_{\mathrm{OBS}}$. Even though the Siberian showed the lowest genetic diversity in general, nucleotide diversity $(\pi)$ was lowest in the Chinese, despite the relative high number of haplotypes and polymorphic sites. These observations are compatible with a recent Han population expansion (Zheng et al., 2011), which would increase the number of haplotypes and polymorphic sites due to the maintenance of new, rare mutations that will have few impact over $\pi$. This is in agreement with the results of neutrality statistics considering that a population expansion would drive these statistics towards negative values. For both Tajima's D (TajD) and Fu's $F_{S}$, the Chinese population is the one with the lowest average scores, even though individual tests are barely statistically significant at $P<0.05$ for TajD and $P<0.02$ for $\mathrm{F}_{\mathrm{S}}$, probably due to the limited power of these tests considering the limited sample size available. On the other hand, for both neutrality statistics, Native Americans have the largest average values, in agreement with a possible genetic bottleneck during the early settlement of the Americas.
Pairwise $\Phi_{\mathrm{ST}}$ values show Siberians closer to the Chinese (Table 2). However, from a locus-by-locus perspective, Siberians are "intermediate" between Chinese and the Native Americans, since for all but one locus Siberians show non-significant $\Phi_{\mathrm{ST}}$ values with one (Chinese, three loci; Native Americans, one loci), or both (four loci) populations (Table S2). Native Americans and Chinese represent the most divergent population pair. This was expected given their more distant geographic relationship and considering recent models for the peopling of the Americas that suggest that the ancestral population of Native Americans had ancestry from both East and West Eurasians (Raghavan et al., 2014). In addition, some sort of secondary genetic contact between Native American and Siberian populations may help to explain the observed pattern (e.g., González-José et al., 2008; Azevedo et al., 2011; Ray et al., 2010; Reich et al., 2012; Raghavan et al., 2015). AMOVA results are very similar irrespective of considering three (Chinese vs. Siberian vs. Native American) or two (Chinese + Siberian vs. Native American) populations, with the among population component explaining $18.13 \%$ or $19.80 \%$ of the total genetic variance, respectively.

\section{Mutation and recombination}

For all nine loci, the constant population size model provided a better fit to the results (data not shown) and, therefore, this model was used for the estimation of the overall evolutionary rate. Substitution rates per site for each locus are presented in (Table S3), and varied from $5.59 \times 10^{-10}$ substitutions/site/year $(\mathrm{s} / \mathrm{s} / \mathrm{y})$ to $1.38 \times 10^{-9} \mathrm{~s} / \mathrm{s} / \mathrm{y}$, with an average value of $9.61 \times 10^{-10} \mathrm{~s} / \mathrm{s} / \mathrm{y}$, which is close to

Table 2 - Average pairwise $\Phi_{\mathrm{ST}}$ (lower diagonal) and their standard deviations over loci (upper diagonal).

\begin{tabular}{lcccc}
\hline Population & Asian & Chinese & Siberian & Native American \\
\hline Asian & - & 0.0198 & 0.1108 & 0.2039 \\
Chinese & -0.0128 & - & 0.1791 & 0.2315 \\
Siberian & 0.0199 & 0.0713 & - & 0.2119 \\
Native American & 0.1963 & 0.2284 & 0.1641 & - \\
\hline
\end{tabular}

Table 1 - Average genetic diversity statistics over loci. Standard deviation values are shown in parentheses and are calculated over loci ${ }^{1}$.

\begin{tabular}{lccccccccc}
\hline Population & $\begin{array}{c}\text { Average no. } \\
\text { hapl. }\end{array}$ & Total S & $\mathrm{S}$ & Gene div. & $H_{\mathrm{OBS}}$ & $H_{\mathrm{EXP}}$ & $\pi(\%)$ & Taj D & Fu's F \\
\hline Asian & $7.33(3.43)$ & 62 & $6.89(3.26)$ & $0.638(0.218)$ & $0.529(0.205)$ & $0.717(0.187)$ & $0.072(0.029)$ & $0.295(1.049)$ & $-0.919(2.825)$ \\
Chinese & $5.44(2.40)$ & 49 & $5.44(2.83)$ & $0.544(0.233)$ & $0.514(0.255)$ & $0.586(0.180)$ & $0.054(0.027)$ & $0.062(1.207)$ & $-0.528(1.920)$ \\
Siberian & $3.67(1.67)$ & 36 & $4.00(2.50)$ & $0.536(0.217)$ & $0.556(0.218)$ & $0.679(0.278)$ & $0.075(0.040)$ & $0.444(0.814)$ & $0.706(1.032)$ \\
Native & $3.78(1.09)$ & 39 & $4.33(2.18)$ & $0.599(0.097)$ & $0.502(0.153)$ & $0.801(0.163)$ & $0.077(0.029)$ & $0.616(0.926)$ & $1.081(1.634)$ \\
American & & & & & & & & & \\
Overall & $8.22(3.83)$ & 66 & $7.33(3.46)$ & $0.674(0.182)$ & $0.521(0.154)$ & $0.790(0.162)$ & $0.082(0.023)$ & $0.743(1.077)$ & $-0.630(3.520)$ \\
\hline
\end{tabular}

Note: ${ }^{1}$ Average no. hapl: average number of haplotypes; total S: total number of S over loci; S: segregating sites; Gene div.: Gene diversity; $H_{\mathrm{OBS}}$ : Ob-

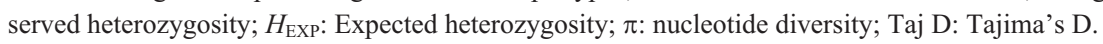


previous estimates for autosomes (Fagundes et al., 2007). For all loci, the null hypothesis of no recombination could not be rejected $(P>0.05$; Table $\mathrm{S} 1)$. These results suggest that eventual recombination events, if any, affecting this dataset have been weak enough to violate the assumption of no recombination among independent loci in the IM models.

\section{IM scenarios}

Results for IM scenarios for different population pairs are presented in Table 3. In general, the effective size of the ancestral population $\left(\mathrm{N}_{\mathrm{A}}\right)$ were estimated within a narrow credible interval in all comparisons. However, most parameters for current effective population sizes had broad credible intervals (Figures S1-S4). Including or not migration resulted in very similar estimates for all parameters (Table 3; Figures S1-S11), and thus we will only discuss the results based on the "full migration" scenarios. Importantly, even though our data is not informative for precise estimates of the migration parameters directly, resulting in flat posterior densities (Figure S5), maintaining migration in the analysis allows estimating the effective size of the founder population of the Americas while accounting for the impact and uncertainty of gene flow estimates (González-José et al., 2008; Azevedo et al., 2011; Ray et al., 2010; Reich et al., 2012; Raghavan et al., 2015; Skoglund et al., 2015).

Divergence time estimates showed two distinct patterns (Figure 1; Figure S6). Whenever Siberians are included in the comparison against Native Americans (either as a single population or as part of an "Asian" metapopulation), the time of divergence goes toward the lower limit set by the prior at $15 \mathrm{kya}$. Even though the IM model should be able to separate the effects of divergence and migration, the relatively small sample sizes and the overall ge-

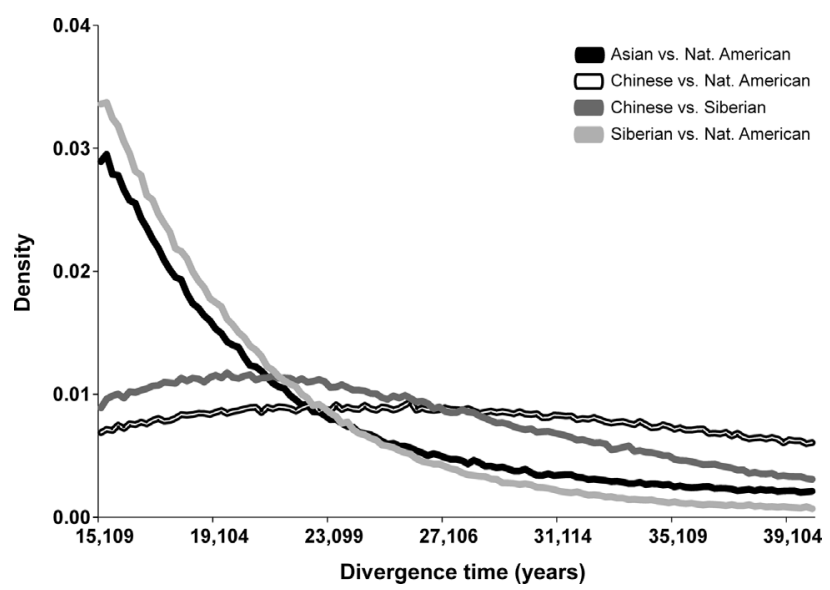

Figure 1 - Posterior densities for divergence time (years) in all scenarios tested including migration. Divergence between Asian and Native American is shown in solid black, between Chinese and Native American in white with black contour, between Chinese and Siberian in dark gray, and between Siberian vs. Native American in light gray, as shown in the graphical legend.

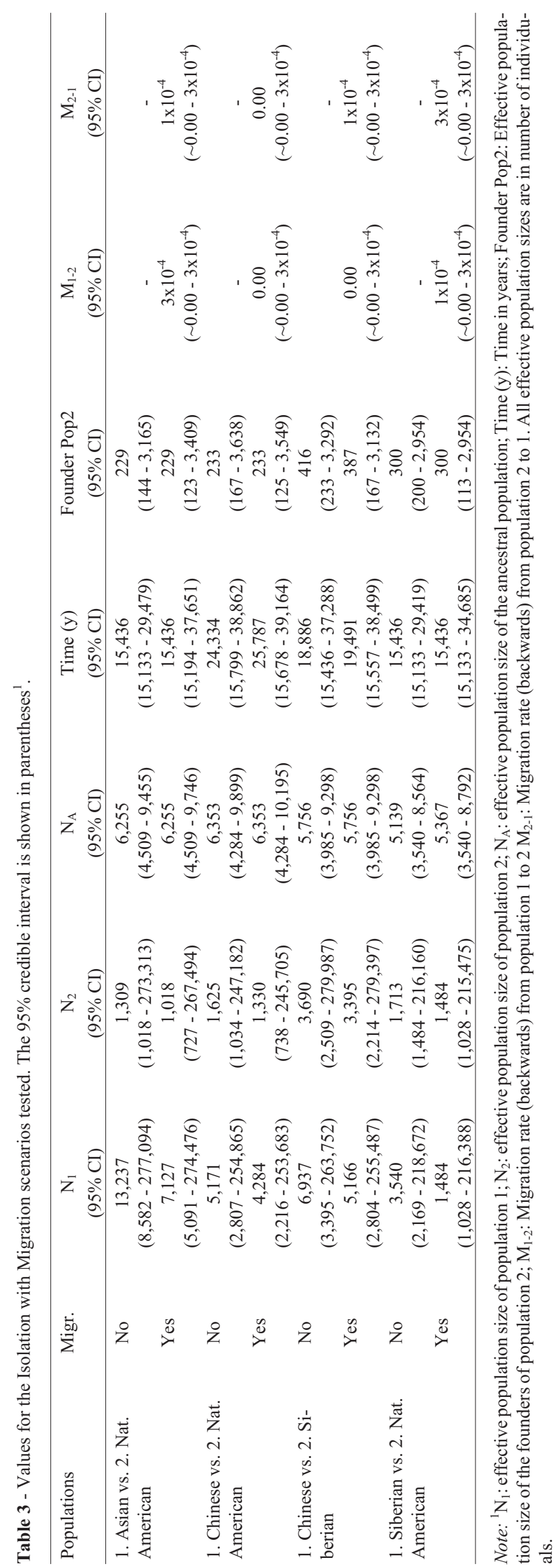


netic similarity between these groups make difficult distinguishing between recent migration and shared ancestry. Alternatively, this result may reflect a genuine impact of recent migration between these groups, even though recent migration is thought as having a weaker impact on Central and South Amerindians compared to North Amerindians (González-José et al., 2008; Azevedo et al., 2011; Ray et al., 2010; Reich et al., 2012; Raghavan et al., 2015). On the other hand, whenever Chinese are contrasted with Native Americans or Siberians the divergence time parameter shows roughly flat posteriors, with point estimates around 25 kya or 19 kya $v s$. Native Americans or Siberians, respectively, in agreement with a more recent shared ancestry between Siberians and Native Americans (Raghavan et al., 2015) compared to Han, even though contemporary Siberians lack the Western Eurasian ancestry component represented by the Mal'ta individual (Raghavan et al., 2014).

Estimates of the splitting parameter $s$ resulted in heavier densities around small values, suggesting, for all scenarios, a reduction on Native American effective population size compared with Asian, Chinese and Siberian populations, as well as another population bottleneck for the Siberian population when compared against the Chinese (Figure S7). The effective population size of the founder populations (parameter Founder Pop2 in Table 3) is the product between the effective size of the ancestral population and the splitting parameter $s$. The posterior densities for all scenarios are presented in Figures S8-S11, and are very similar for scenarios including or not migration. Considering scenarios with migration (Figure 2), the effective size of the founding population for Native Americans was estimated around 229 (vs. Asians), 233 (vs. Chinese), or 300 individuals (vs. Siberian), with $95 \%$ credible intervals between $\sim 100-3,700$ individuals. It should be noted, however, that even though the confidence intervals are wide (Table 3), the density is asymmetrical, with much of the

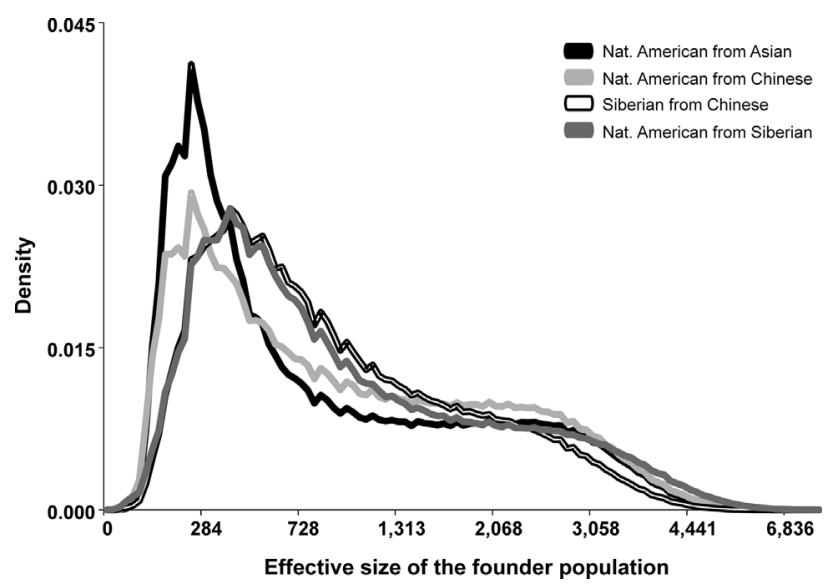

Figure 2 - Posterior densities for the effective sizes of the founder population. The size of the founder population for Native American from Asia is shown in solid black, for Native American from Chinese in light gray, for Siberian from Chinese in white with black contour, and for Native American from Siberian in dark gray, as shown in the graphical legend. posterior probability falling closer to the smallest values (Figure 2). For example, in the case of the Asian vs. Native American comparison, the $50 \%$ highest posterior density falls within a range of small values (between 123-587 individuals), suggesting that small values are more likely than larger ones. These values represent intermediate estimates between the extreme bottleneck scenario proposed by Hey (2005), and the larger numbers estimated by Kitchen et al. (2008). Our results also show some evidence of a genetic bottleneck during the divergence of Siberian populations from their Asian (Chinese) ancestors (Figure 2), but while such reduction may have been milder than that associated to the peopling of the Americas, the credible intervals for these estimates are broad.

Our estimates for the effective size of the founder population of Native (Central and South) Americans are in good agreement with those reported for other autosomal markers (Figure 3), except for the original estimates of Hey (2005) which are smaller, probably due to the use of an unconstrained prior on migration rate, as suggested by Kitchen et al. (2008). On the other hand, estimates including complete mtDNA genomes (Fagundes et al., 2008; Kitchen et al., 2008) are usually larger (Figure 3). This may be due to a larger effective population size for women. Further analysis of X-chromosome and Y-chromosome data would help to clarify this issue. Interestingly, our estimates were comparable with those based on studies using admixed populations from a restricted geographic area $(\mathrm{Gu}-$ tenkunst et al., 2009; Wall et al., 2011; Gravel et al., 2013), suggesting that Latino populations may be extremely valuable sources of information on Native American history, as

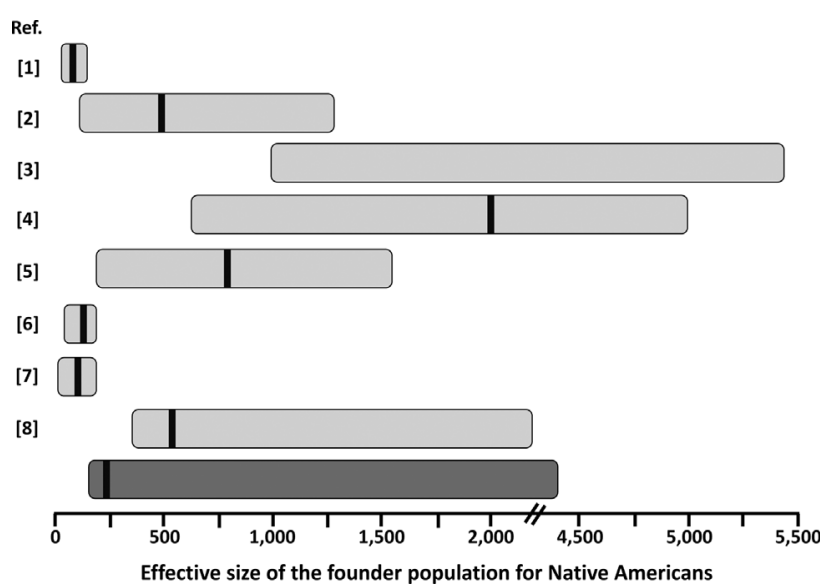

Figure 3 - Schematic representation of estimates of the founder population size for Native Americans from this (dark gray bar) and previous (light gray bars) studies. The horizontal bars represent the approximate 95\% credible interval for the published values. Point estimates, when reported, are represented by the black vertical bar within the horizontal bars. Please note the discontinuity in the $x$-axis between 2,000 and 4,500. References (Ref.) are: 1 - Hey (2005); 2 - Fagundes et al. (2007); 3 - Kitchen et al. (2008); 4 -Fagundes et al. (2008); 5-Gutenkunst et al. (2009); 6 - Ray et al. (2010); 7 - Wall et al. (2011); 8 - Gravel et al. (2013). The values presented in Hey (2005) were recalculated based on a generation time of 25 years. 
have been shown for inferences on extinct Native American ethnicities (Marrero et al., 2007). Estimating the effective size of the Native American founder population is important in medical genetic approaches, as in the case of estimating the average size of linkage disequilibrium blocks and how many genetic markers (e.g. SNPs) will be effective for gene-disease mapping in this (or derived) population (e.g., Wall et al., 2011). This parameter is also of crucial importance to understand the fate of adaptive alleles in the founder population of Native Americans, that might behave as neutral depending on the effective population size (Ohta, 1992). A relatively strong reduction in effective size in the founder population of Native Americans might explain why some possibly adaptive genetic variants in other populations do not show any signature of selection in the Americas (e.g., Paixão-Côrtes et al., 2011; Augusto et al., 2013).

Our study used a relatively small sample size to estimate the effective size of the founder population. Felsenstein (2006) suggested that a small number of individuals (n $\leq 8)$ may be sufficient for estimating the effective population size, because most of each gene genealogy would be known with a limited number of genetic lineages, provided that a sufficient number of independent genealogies were studied. Therefore, this number seems appropriate for a broad characterization of the effective size of the founder population, even though it is certainly very small to thoroughly characterize the genetic diversity of these populations at these loci. Other interesting questions regarding the peopling of the Americas, such as differences in the effective population size for different regions within the New World, would certainly require a much larger sample size. As discussed previously, it is noteworthy that our results provided similar results compared to other autosomalbased studies, even when only local admixed populations were sampled.

One of the major statistical advantages of the IM model is that it can use the full dataset in a maximum likelihood framework, which increase the power of evolutionary demographic parameter estimation compared to techniques such as approximate Bayesian computation (Beaumont, 2008; Nielsen and Beaumont, 2009). Such higher statistical power, however, may come at the expense of some biological realism. For example, scenarios suggesting gene flow between Asia and America usually assume that gene flow had a late start compared to the initial population subdivision (González-José et al., 2008; Ray et al., 2010). Unfortunately it is not possible yet to implement such specific constrains within the full likelihood framework of the IM models (Nielsen and Beaumont, 2009). Interestingly, in the present analysis including or not including migration did not result in major differences for any demographic parameter. Migration would reduce the estimates for the founder population size in the context of the peopling of the New World (Kitchen et al., 2008). This is intuitive, since migra- tion would lead to new genetic diversity coming to the continent, thus resulting in a smaller population size estimate for the initial founding event. The recent discussions on the importance of secondary migration to account for the morphological and genetic diversity of Native Americans (González-José et al., 2008; Azevedo et al., 2011; Ray et al., 2010; Reich et al., 2012; Raghavan et al., 2015; Skoglund et al., 2015; von Cramon-Taubadel et al., 2017) indicate the need to add a further step on the traditional three-stage model (Mulligan and Szathmàry, 2017).

\section{Acknowledgments}

We thank the individuals who agreed to participate in this investigation. We are also thankful to Cladinara $\mathrm{R}$. Sarturi for technical help, and to two anonymous reviewers for comments and corrections in a previous version of this text. This research was supported by Conselho Nacional de Desenvolvimento Científico e Tecnológico (CNPq) research grants (477780/2003-2 to SLB, and 151263/2007-8 to NJRF) and by National Science Foundation grants (OPP-9905090 and OPP-0327676 to MHC).

\section{References}

Achilli A, Perego UA, Bravi CM, Coble MD, Kong QP, Woodward SR, Salas A, Torroni A and Bandelt HJ (2008) The phylogeny of the four pan-American mtDNA haplogroups: implications for evolutionary and disease studies. PLoS One 3:e1764.

Achilli A, Perego UA, Lancioni H, Olivieri A, Gandini F, Kashani BH, Battaglia V, Grugni V, Angerhofer N, Rogers MP, et al. (2013) Reconciling migration models to the Americas with the variation of North American native mitogenomes. Proc Natl Acad Sci U S A 110:14308-14313.

Augusto DG, Piovezan BZ, Tsuneto LT, Callegari-Jacques SM and Petzl-Erler ML (2013) KIR gene content in Amerindians indicates influence of demographic factors. PLoS One 8:e56755.

Azevedo S, Nocera A, Paschetta C, Castillo L, González M and González-José R (2011) Evaluating microevolutionary models for the early settlement of the New World: the importance of recurrent gene flow with Asia. Am J Phys Anthropol 146:539-552.

Beaumont MA (2008) Joint determination of topology, divergence time, and immigration in population trees. In: Matsumura S, Forster P and Renfrew C (eds) Simulation, Genetics, and Human Prehistory. McDonald Institute for Archaeological Research, Cambridge, pp 135-154.

Bisso-Machado R, Jota MS, Ramallo V, Paixão-Côrtes VR, Lacerda DR, Salzano FM, Bonatto SL, Santos FR and Bortolini MC (2011) Distribution of Y-chromosome Q lineages in Native Americans. Am J Hum Biol 23:563-566.

Bisso-Machado R, Bortolini MC and Salzano FM (2012) Uniparental genetic markers in South Amerindians. Genet Mol Biol 35:365-387.

Charlesworth B (2009) Effective population size and patterns of molecular evolution and variation. Nat Rev Genet 10:195205. 
Drummond AJ and Rambaut A (2007) BEAST: Bayesian evolutionary analysis by sampling trees. BMC Evol Biol 7:214.

Drummond AJ, Rambaut A, Shapiro B and Pybus OG (2005) Bayesian coalescent inference of past population dynamics from molecular sequences. Mol Biol Evol 22:1185-1192.

Drummond AJ, Ho SYW, Phillips MJ and Rambaut A (2006) Relaxed phylogenetics and dating with confidence. PLoS Biology $4: \mathrm{e} 88$.

Dunn IS and Blattner FR (1987) Sharons 36 to 40: Multi-enzyme, high capacity, recombination deficient replacement vectors with polylinkers and polystuffers. Nucleic Acids Res 15:2677-2698.

El Sharawy A, Warner J, Olson J, Forster M, Schilhabel MB, Link DR, Rose-John S, Schreiber S, Rosenstiel P, Brayer J, et al. (2012) Accurate variant detection across non-amplified and whole genome amplified DNA using targeted next generation sequencing. BMC Genomics 13:500.

Ewing B, Hillier L, Wendl MC and Green P (1998) Base-calling of automated sequencer traces using phred. I. Accuracy assessment. Genome Res 8:175-185.

Excoffier L and Lischer HE (2010) Arlequin suite ver 3.5: A new series of programs to perform population genetics analyses under Linux and Windows. Mol Ecol Res 10:564-567.

Fagundes NJR, Ray N, Beaumont M, Neuenschwander S, Salzano FM, Bonatto SL and Excoffier L (2007) Statistical evaluation of alternative models of human evolution. Proc Natl Acad Sci U S A 104:17614-17619.

Fagundes NJR, Kanitz R, Eckert R, Valls ACS, Bogo MR, Salzano FM, Smith DG, Silva Jr WA, Zago MA, Ribeiro-dosSantos AK, et al. (2008) Mitochondrial population genomics supports a single pre-Clovis origin with a coastal route for the peopling of the Americas. Am J Hum Genet 82:583592.

Felsenstein J (2006) Accuracy of coalescent likelihood estimates: Do we need more sites, more sequences, or more loci? Mol Biol Evol 23:691-700.

Frisse L, Hudson RR, Bartoszewicz A, Wall JD, Donfack J and Di Rienzo A (2001) Gene conversion and different population histories may explain the contrast between polymorphism and linkage disequilibrium levels. Am J Hum Genet 69:831-843.

Goebel T, Waters MR and O'Rourke DH (2008) The Late Pleistocene dispersal of modern humans in the Americas. Science 319:1497-1502.

González-José R and Bortolini MC (2011) Integrating different biological evidence around some microevolutionary processes: bottlenecks and Asian-American Arctic gene flow in the New World settlement. Evol Edu Outreach 4:232-243.

González-José R, Bortolini MC, Santos FR and Bonatto SL (2008) The peopling of America: Craniofacial shape variation on a continental scale and its interpretation from an interdisciplinary view. Am J Phys Anthropol 137:175-187.

Gordon D, Abajian C and Green P (1998) Consed: A graphical tool for sequence finishing. Genome Res 8:195-202.

Gravel S, Zakharia F, Moreno-Estrada A, Byrnes JK, Muzzio M, Rodriguez-Flores JL, Kenny EE, Gignoux CR, Maples BK, Guiblet W, et al. (2013) Reconstructing Native American migrations from whole-genome and whole-exome data. PLoS Genet 9:e1004023.
Greenberg JH, Turner CG and Zegura SL (1986) The settlement of the Americas: A comparison of the linguistic, dental, and genetic evidence. Curr Anthropol 27:477-497.

Gutenkunst RN, Hernandez RD, Williamson SH and Bustamante CD (2009) Inferring the joint demographic history of multiple populations from multidimensional SNP frequency data. PLoS Genet 5:e1000695.

Hartl DL and Clark AG (2007) Principles of Population Genetics. 4th edition. Sinauer Associates, Sunderland, $565 \mathrm{p}$.

Hey J (2005) On the number of New World founders: A population genetic portrait of the peopling of the Americas. PLoS Biology 3:e193.

Kass RE and Raftery AE (1995) Bayes factors. J Am Stat Assoc 90:773-795.

Kitchen A, Miyamoto MM and Mulligan CJ (2008) A three-stage colonization model for the peopling of the Americas. PLoS One 3:e1596.

Li JZ, Absher DM, Tang H, Southwick AM, Casto AM, Ramachandran S, Cann HM, Barsh GS, Feldman M, CavalliSforza LL, et al. (2008) Worldwide human relationships inferred from genome-wide patterns of variation. Science 319:1100-1104.

Librado P and Rozas J (2009) DnaSP v5: A software for comprehensive analysis of DNA polymorphism data. Bioinformatics 25:1451-1452.

Long JC, Li J and Healy ME (2009) Human DNA sequences: More variation and less race. Am J Phys Anthropol 139:23-34.

Macaulay V, Hill C, Achilli A, Rengo C, Clarke D, Meehan W, Blackburn J, Semino O, Scozzari R, Cruciani F, et al. (2005) Single, rapid coastal settlement of Asia revealed by analysis of complete mitochondrial genomes. Science 308:10341036.

Marangoni A, Caramelli D and Manzi G (2014) Homo sapiens in the Americas. Overview of the earliest human expansion in the New World. J Anthropol Sci 92:79-97.

Marrero AR, Bravi C, Stuart S, Long JC, Leite FPN, Kommers T, Carvalho CM, Pena SD, Ruiz-Linares A, Salzano FM, et al. (2007) Pre- and post-Columbian gene and cultural continuity: The case of the Gaucho from southern Brazil. Hum Hered 64:160-171.

Mulligan CJ and Szathmáry EJ (2017) The peopling of the Americas and the origin of the Beringian occupation model. Am J Phys Anthropol 162:403-408.

Mulligan CJ, Kitchen A and Miyamoto MM (2008) Updated three-stage model for the peopling of the Americas. PLoS One 3:e3199.

Nielsen R and Beaumont MA (2009) Statistical inferences in phylogeography. Mol Ecol 18:1034-1047.

Ohta T (1992) The nearly neutral theory of molecular evolution. Annu Rev Ecol Syst 23:263-286.

O'Rourke DH (2011) Contradictions and concordances in American colonization models. Evol Edu Outreach 4:244-253.

Paixão-Côrtes VR, Meyer D, Pereira TV, Mazières S, Elion J, Krishnamoorthy R, Zago MA, Silva Jr WA, Salzano FM and Bortolini MC (2011) Genetic variation among major human geographic groups supports a peculiar evolutionary trend in PAX9. PLoS One 6:e15656.

Prugnolle F, Manica A and Balloux F (2005) Geography predicts neutral genetic diversity of human populations. Curr Biol 15:R159-160. 
Raghavan M, Skoglund P, Graf KE, Metspalu M, Albrechtsen A, Moltke I, Rasmussen S, Stafford TW, Orlando L, Metspalu E, et al. (2014) Upper Palaeolithic Siberian genome reveals dual ancestry of Native Americans. Nature 505:87-91.

Raghavan M, Steinrücken M, Harris K, Schiffels S, Rasmussen S, DeGiorgio M, Albrechtsen A, Valdiosera C, Ávila-Arcos MC, Malaspinas AS, et al. (2015) Genomic evidence for the Pleistocene and recent population history of Native Americans. Science 349:aab3884.

Rasmussen M, Sikora M, Albrechtsen A, Korneliussen TS, Moreno-Mayar JV, Poznik GD, Zollikofer CP, Ponce de León MS, Allentoft ME, Moltke I, et al. (2015) The ancestry and affiliations of Kennewick Man. Nature 523:455-458.

Ray N, Wegmann D, Fagundes NJR, Wang S, Ruiz-Linares A and Excoffier L (2010) A statistical evaluation of models for the initial settlement of the American continent emphasizes the importance of gene flow with Asia. Mol Biol Evol 27:337345.

Reich D, Patterson N, Campbell D, Tandon A, Mazieres S, Ray N, Parra MV, Rojas W, Duque C, Mesa N, et al. (2012) Reconstructing Native American population history. Nature 488:370-374.

Rozas J, Gullaud M, Blandin G and Aguade M (2001) DNA variation at the rp 49 gene region of Drosophila simulans: Evolutionary inferences from an unusual haplotype structure. Genetics 158:1147-1155.

Salzano FM (2007) The prehistoric colonization of the Americas. In: Crawford MH (ed) Anthropological Genetics: Theory, Methods, and Applications. Cambridge University Press, Cambridge, pp 433-455.

Scliar MO, Soares-Souza GB, Chevitarese J, Lemos L, Magalhães WC, Fagundes NJR, Bonatto SL, Yeager M, Chanock SJ and Tarazona-Santos E (2012) The population genetics of Quechuas, the largest native South American group: Autosomal sequences, SNPs, and microsatellites evidence high level of diversity. Am J Phys Anthropol 147:443-451.

Skoglund P, Mallick S, Bortolini MC, Chennagiri N, Hünemeier T, Petzl-Erler ML, Salzano FM, Patterson N and Reich D (2015) Genetic evidence for two founding populations of the Americas. Nature 525:104-108.

Stephens M, Smith N and Donnelly P (2001) A new statistical method for haplotype reconstruction from population data. Am J Hum Genet 68:978-989.

Tamm E, Kivisild T, Reidla M, Metspalu M, Smith DG, Mulligan CJ, Bravi CM, Rickards O, Martinez-Labarga C, Khusnutdinova EK, et al. (2007) Beringian standstill and spread of Native American founders. PLoS One 2:e829.

Voight BF, Adams AM, Frisse LA, Qian Y, Hudson RR and Di Rienzo A (2005) Interrogating multiple aspects of variation in a full resequencing data set to infer human population size changes. Proc Natl Acad Sci U S A 102:18508-18513.

von Cramon-Taubadel N, Strauss A and Hubbe M (2017) Evolutionary population history of early Paleoamerican cranial morphology. Sci Adv 3:e1602289.

Wall JD, Cox MP, Mendez FL, Woerner A, Severson T and Hammer MF (2008) A novel DNA sequence database for analyzing human demographic history. Genome Res 18:13541361.
Wall JD, Jiang R, Gignoux C, Chen GK, Eng C, Huntsman S and Marjoram P (2011) Genetic variation in Native Americans, inferred from Latino SNP and resequencing data. Mol Biol Evol 28:2231-2237.

Yu N, Chen FC, Ota S, Jorde LB, Pamilo P, Patthy L, Ramsay M, Jenkins T, Shyue SK and Li WH (2002) Larger genetic differences within Africans than between Africans and Eurasians. Genetics 161:269-274.

Zegura SL, Karafet TM, Zhivotovsky LA and Hammer MF (2004) High-resolution SNPs and microsatellite haplotypes point to a single, recent entry of Native American Y chromosomes into the Americas. Mol Biol Evol 21:164-175.

Zheng H-X, Yan S, Qin Z-D, Wang Y, Tan J-Z, Li H and Jin L (2011) Major population expansion of East Asians began before Neolithic time: evidence of mtDNA genomes. PLoS One 6:e25835.

\section{Supplementary material}

The following online material is available for this article: Table S1 - Genetic diversity statistics for each locus.

Table S2 - Pairwise $\Phi_{\mathrm{ST}}$ for each locus.

Table S3 - Estimated substitution rates $(\mu)$ for each locus. Figure S1 - Posterior density of effective population sizes for Asian vs. Native Americans.

Figure S2 - Posterior density of effective population sizes for Chinese vs. Native Americans.

Figure S3 - Posterior density of effective population sizes for Chinese vs. Siberians.

Figure S4 - Posterior density of effective population sizes for Siberian vs. Native Americans.

Figure S5 - Posterior density for migration parameters for all scenarios.

Figure S6 - Posterior density for divergence times (in years) for all scenarios.

Figure S7 - Posterior density for the splitting parameter s for all scenarios.

Figure S8 - Posterior density for the effective size of the founder population for Native Americans, from Asia, with or without migration.

Figure S9 - Posterior density for the effective size of the founder population for Native Americans, from Chinese, with or without migration.

Figure S10 - Posterior density for the effective size of the founder population for Siberians, from Chinese, with or without migration.

Figure S11 - Posterior density for the effective size of the founder population for Native Americans, from Siberians, with or without migration.

Associate Editor: Loreta B. Freitas

License information: This is an open-access article distributed under the terms of the Creative Commons Attribution License (type CC-BY), which permits unrestricted use, distribution and reproduction in any medium, provided the original article is properly cited. 